\title{
Formal Electrophilic Phenylation Reaction with Tropylium Ion
}

\author{
Demelza J. M. Lyons, ${ }^{\S}$ An H. Dinh, ${ }^{\S}$ Reece D. Crocker, ${ }^{\S}$ Binh Khanh Mai, ${ }^{\dagger}, *$ Thanh Vinh Nguyen ${ }^{\S, *}$
}

${ }^{+}$School of Chemistry, University of New South Wales, Sydney, NSW 2052, Australia

${ }^{\S}$ Department of Chemistry, University of Pittsburgh, Pittsburgh, Pennsylvania 15260, United States

Tropylium ion as a formal electrophilic Ph building block

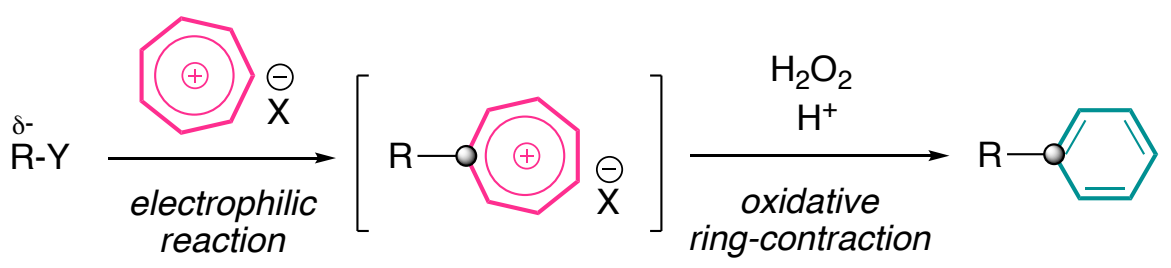

Abstract: Arylation reaction is an important transformation in synthetic chemistry as aryl building blocks are ubiquitous in valuable organic frameworks. Traditionally, this type of reaction has been carried out either via biaryl coupling reactions or with the use of reactive intermediates such as arynes or aryl radicals. Direct electrophilic arylation reactions have been rarely reported in literature, as the required arenium building blocks are often unstable or inaccessible. To develop a new strategy for such transformation, we herein introduce the development of a formal phenylation reaction, which proceeds via an electrophilic cycloheptatrienylation with tropylium ion, followed by an oxidative ring-contraction. 
As aryl moieties are ubiquitous in many synthetic organic structures and biologically valuable compounds, installation of aryl substituents becomes one of the most frequently used chemical transformations in organic chemistry. ${ }^{1}$ There have been numerous approaches for arylation reactions, most notably transition metal-catalyzed coupling methods such as the Ullmann, ${ }^{2} \mathrm{Kumada}^{3}$ Negishi, ${ }^{4}$ Stille, ${ }^{5}$ Suzuki-Miyaura, ${ }^{6}$ Hiyama, ${ }^{7}$ and other reactions. ${ }^{8}$ Due to their excellent efficiency and broad scope, these reactions have been extensively used for biaryl couplings or arylation of aliphatic substrates in organic syntheses. ${ }^{8}$ However, they are not without drawbacks, which include the use of specifically designed precursors and precious transition-metal catalysts, highly toxic reagents, or harsh reaction conditions. ${ }^{8}$ Transition-metal residues remaining in target products also pose significant interference to their downstream synthetic or biological applications. ${ }^{9}$ These issues led to recent efforts to develop transition metal-free aryl-aryl coupling methods, many of them are however with disputable outcomes. ${ }^{10}$

For phenylation reactions in particular, it is possible to avoid transition metal coupling chemistry by treating substrates with highly reactive phenylating reagents such as benzyne ${ }^{11}$ or phenyl radical, which can be generated from phenyldiazonium or phenyliodonium salts (Scheme 1). ${ }^{12}$ However, the synthetic precursors of these reactive intermediates normally require lengthy synthetic sequences to prepare; and their high reactivity often leads to unwanted side reactions. It is therefore of great interest to develop a new synthetic paradigm for the phenylation reaction.

(a) Cycloheptatrienes as carbene precursors

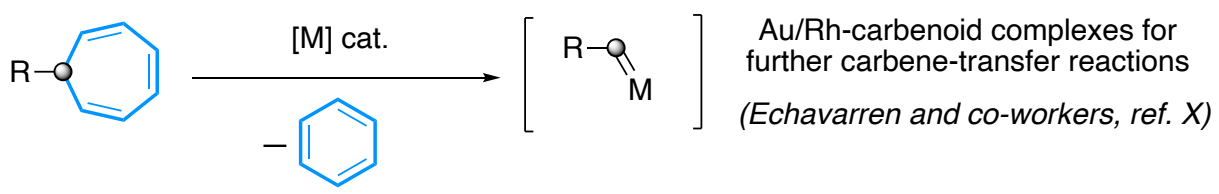

(b) THIS WORK: Tropylium ion as a formal electrophilic Ph building block
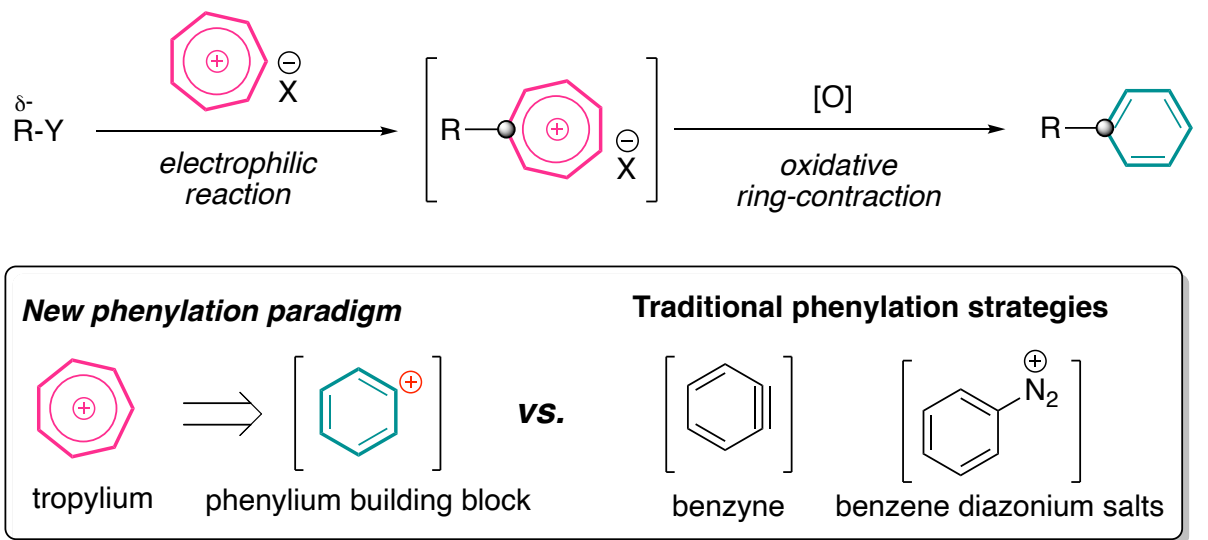

Scheme 1. Tropylium as an electrophilic phenyl building block. 
During the course of our recent investigations on the synthetic utility of the non-benzenoid aromatic tropylium ion, ${ }^{13}$ we found that it can efficiently couple with a broad range of nucleophilic substrates such as alkenes, arenes, or organometallic reagents to form cycloheptatriene derivatives. The cycloheptatriene moiety can also be easily converted to a tropylium ion again by simple hydride abstraction. We believe that an oxidative ring contraction reaction ${ }^{14}$ can be performed on the electron deficient seven-membered ring of the tropylium $\left(-\mathrm{C}_{7} \mathrm{H}_{6}{ }^{+}\right)$ion to transform it into a phenyl ring $\left(-\mathrm{C}_{6} \mathrm{H}_{5}\right)$. The cleavage of one carbon from the seven-membered ring, ${ }^{15}$ to retain a phenyl group on the original organic framework, is directly opposite but complementary to the elegant cycloheptatriene chemistry developed by the Echavarren group, in which they use $\mathrm{Au}(\mathrm{I})$ or $\mathrm{Rh}(\mathrm{II})$ catalysis to eliminate a benzene ring from cycloheptatriene derivatives to produce organometallic carbenoid complexes for further carbene-transfer cycloaddition and insertion reactions (Scheme 1a). ${ }^{16}$

Our approach would allow a novel strategy to formally install phenyl group in an electrophilic fashion without transition metal catalysis, which will have tremendous potential in synthetic chemistry. It is also an interesting transformation at fundamental level that the non-benzenoid aromatic tropylium ring is converted to the aromatic benzene ring. Our calculations of the nucleus-independent chemical shifts $\left(\mathrm{NICS}(1)_{\mathrm{zz}}\right)$ values $^{17}$ for tropylium ion and benzene are -27.7 and $-30.8 \mathrm{ppm},{ }^{18}$ respectively, which indicate that benzene ring has higher aromaticity than tropylium ion. Hence the ring contraction transformation from a tropylium ion to a phenyl group should be energetically favorable. Herein, we report the development of an experimental protocol to couple nucleophiles with the tropylium ion. This electrophilic building block was then subjected to an oxidative ring-contraction to afford a formal phenylation process (Scheme 1b).

We started our investigation of this formal phenylation reaction by screening the reaction conditions to ring contract phenyl tropylium tetrafluoroborate $1 \mathbf{a}$ into biphenyl $\mathbf{2 a}$ in an oxidizing environment, as the tropylium moiety is electrophilic. After an extensive optimization study, ${ }^{18}$ we established that the reaction was best carried out in aqueous/acetonitrile environment with $\mathrm{H}_{2} \mathrm{O}_{2}$ (3 equiv.) as the oxidant in the presence of $\mathrm{HBF}_{4}$ (2 equiv.) to give the product in excellent yield of $93 \%$ (Table 1). The use of less $\mathrm{HBF}_{4}$ or a different Brønsted acid led to lower product yields. Similarly, replacing $\mathrm{H}_{2} \mathrm{O}_{2}$ with other commonly used oxidants such as Oxone ${ }^{\circledR},\left(\mathrm{NH}_{4}\right)_{2} \mathrm{Ce}\left(\mathrm{NO}_{3}\right)_{6},{ }^{t} \mathrm{BuOOH}$ or even bleach also resulted in poorer efficiencies. ${ }^{18}$ 
Table 1. Optimization of the oxidative ring-contraction.

\section{Optimal conditions:}

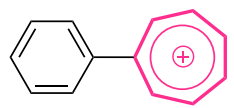

1a $\Theta \mathrm{BF}_{4}$
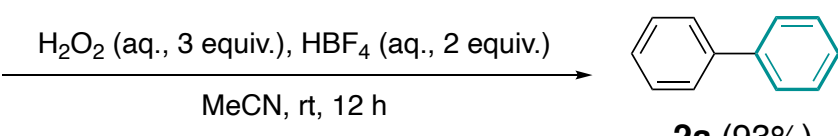

2a $(93 \%)$

\begin{tabular}{ccc}
\hline [a] & Variations from optimal conditions ${ }^{[b]}$ & Yield $^{[c]}$ \\
\hline 1 & no HBF$_{4}$ & $56 \%$ \\
2 & $\mathrm{H}_{2} \mathrm{O}_{2}$ (1 equiv.), no $\mathrm{HBF}_{4}$ & $28 \%$ \\
3 & only 1.0 equiv. of $\mathrm{HBF}_{4}$ & $81 \%$ \\
4 & $\mathrm{H}_{2} \mathrm{SO}_{4}$ (2 equiv.) instead of $\mathrm{HBF}_{4}$ & $52 \%$ \\
5 & $\mathrm{Oxone}^{\circledR}\left(3\right.$ equiv.) instead of $\mathrm{H}_{2} \mathrm{O}_{2}$ & $52 \%$ \\
6 & $\mathrm{Oxone}{ }^{\circledR}$ (3 equiv.), no $\mathrm{HBF}_{4}$ & $59 \%$ \\
7 & $\mathrm{CAN}\left(3\right.$ equiv.) instead of $\mathrm{H}_{2} \mathrm{O}_{2}$ & $31 \%$ \\
8 & ${ }^{t} \mathrm{BuOOH}\left(3\right.$ equiv.) instead of $\mathrm{H}_{2} \mathrm{O}_{2}$ & $69 \%$
\end{tabular}

[a] Conditions: 1a $(0.5 \mathrm{mmol})$, acid and oxidant in $\mathrm{MeCN}(2 \mathrm{~mL})$ under ambient conditions at room temperature. [b] For further experiments on optimization studies, see page S28 in the experimental SI. [c] Yield of the isolated product $2 \mathbf{a}$.

We were curious to understand the mechanistic insights of these reactions, with the hope that they will lead to direct synthetic applications for this reaction as well as inform future developments in chemistry of non-traditional aromatic compounds. However, we were met with little success in our attempts to trap reaction intermediates in the conversion of $\mathbf{1 a}$ to $\mathbf{2} \mathbf{a}$, as the reaction was in partially aqueous and oxidative environment. Thus, density functional theory (DFT) calculations ${ }^{19}$ were carried out to locate a plausible pathway for this oxidative ring contraction. The computational studies were initiated by locating transition states for the reaction between the tropylium salt 1a (Scheme 2) and neutral hydrogen peroxide $\mathrm{H}_{2} \mathrm{O}_{2}$. However, all transition states that we could locate are calculated to associate with very high activation barriers (> $30 \mathrm{kcal} / \mathrm{mol}$, see Figure S1 in the computational SI). 


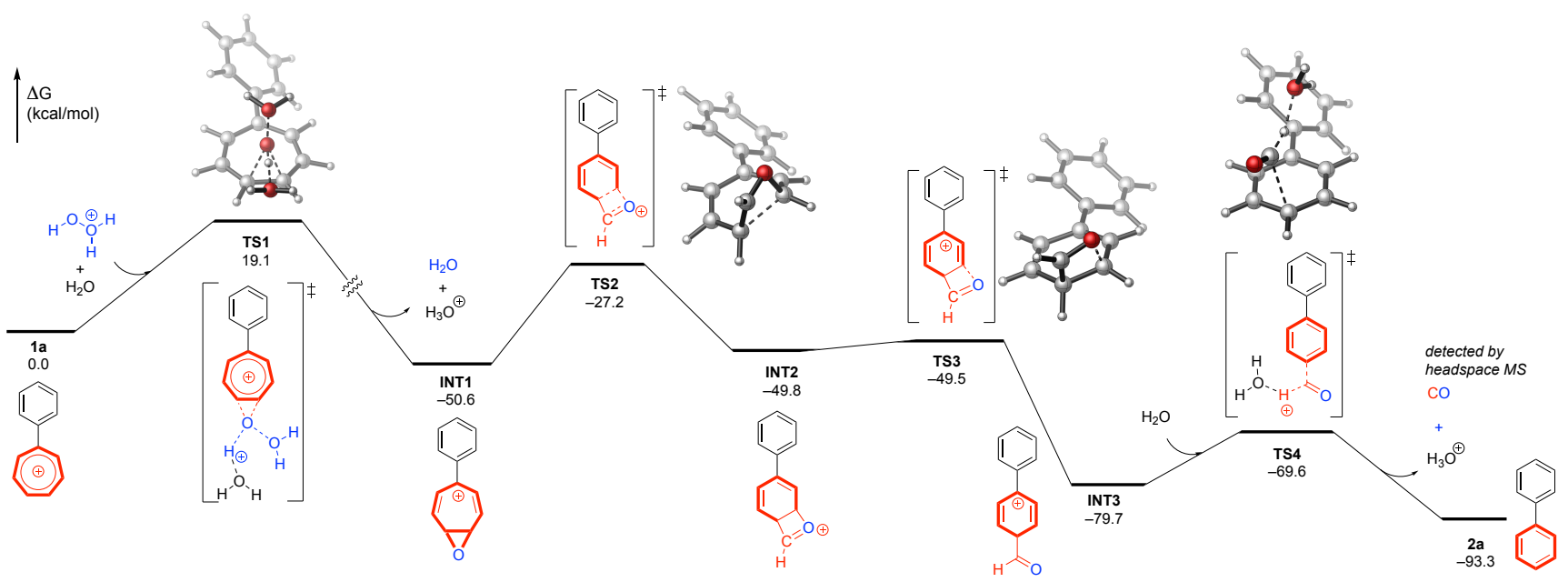

Scheme 2. Computational mechanistic elucidation of the oxidative ring-contraction reaction.

This result is inconsistent with the experimental finding in Table 1 where we found that the reaction can occur efficiently at ambient temperature, albeit in a strongly acidic environment. We then carried out calculations with the assumption that under these reaction conditions, $\mathrm{H}_{2} \mathrm{O}_{2}$ is protonated by fluoroboric acid to generate a highly reactive species $\mathrm{HOOH}_{2}{ }^{+20}$, which indeed led to a feasible reaction pathway. The computed free energy profile and optimized structures of transition states for the reaction between the tropylium ion 1a and the protonated hydrogen peroxide $\mathrm{HOOH}_{2}{ }^{+}$ are shown in Scheme 2. The reaction starts with the electrophilic addition of the $\mathrm{HOOH}_{2}^{+}$species to tropylium ion 1a via transition state TS1, giving oxirane intermediate INT1. The activation energy of TS1 is calculated to be $19.1 \mathrm{kcal} / \mathrm{mol}$ relative to $1 \mathrm{a}$. The feasible barrier of TS1 is primarily initiated by the relatively low energy of LUMO of $\mathrm{HOOH}_{2}{ }^{+20 a}$

To proceed, calculations suggested that skeletal rearrangements ${ }^{21}$ via transition states TS2 and TS3 take place, generating cyclohexadienylium intermediate INT3. Subsequent decomposition of INT3 can then occur via TS4, giving product $2 a$ and $\mathrm{CO}$. The formation of CO molecule $(M=28)$ from the reaction mixture was detected by headspace mass-spectrometry, supporting this proposed mechanism of our oxidative ring contraction reaction. Our DFT calculations show that the ratedetermining step is TS2 with an overall barrier of $23.5 \mathrm{kcal} / \mathrm{mol}$ (Scheme 2). This energy barrier is consistent with our mild reaction conditions. The overall reaction is calculated to be exergonic by $93.3 \mathrm{kcal} / \mathrm{mol}$, which explains why the reaction can proceed to transform the non-benzenoid aromatic tropylium ion into the aromatic benzene ring.

Having the mechanistic insights of the tropylium moiety to the phenyl ring (Scheme 2) and the optimal conditions of the oxidative ring-contraction (Table 1) in hand, we subsequently applied this 
reaction to a synthetic sequence to formally introduce the phenyl group onto a range of aryl halide, alkyl halide and arene substrates (3 or $\mathbf{4}$, Table 2). The synthetic sequence started with the electrophilic cycloheptatrienylation of the organometallic reagents derived from halides $\mathbf{3}$ to form intermediates 6 , which is a typical procedure to make cycloheptatrienyl derivatives. ${ }^{16 a}$ Electron-rich arenes $\mathbf{4}$ could also be directly cycloheptatrienylated via the Friedel-Craft type reaction between them and tropylium tetrafluoroborate 5 a (Table 2$){ }^{13 p}$

Table 2. Substrate scope of the formal phenylation reaction. ${ }^{[a]}$

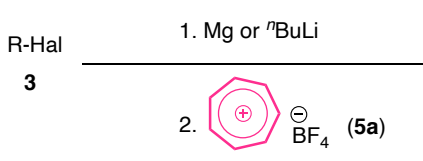

electrophilic cycloheptatrienylation

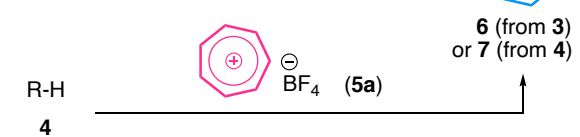

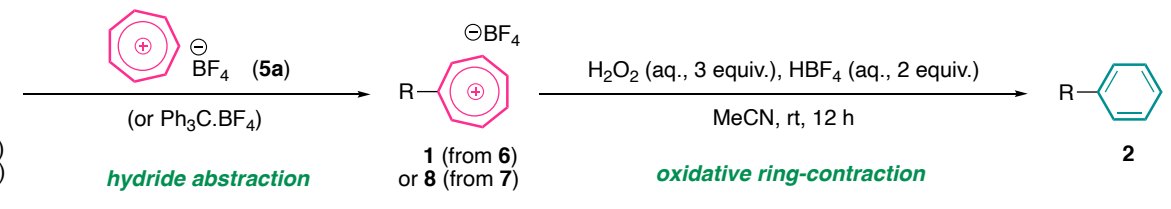

(c)

[a] Further details about the synthesis of 1, 6, 7, and $\mathbf{8}$ can be found in the experimental SI. [b] Yield of the isolated product from the previous precursor in the synthetic sequence.

Cycloheptatrienes 6 or $\mathbf{7}$ were then subjected to a hydride abstraction reaction ${ }^{13 p}$ with tropylium or tritylium salts to turn them into aryl tropylium salts $\mathbf{1}$ or $\mathbf{8}$. Subsequently, $\mathbf{1}$ or $\mathbf{8}$ were oxidized using 
our optimal conditions developed in Table 1 to obtain the formal phenylated products 2 . Yields of isolated intermediates and products for each synthetic step are documented in Table 2. We could also carry out direct a-cycloheptatrienylation reactions on a range of ketones and ketoesters (4o-v). Unfortunately, this cycloheptatriene moiety on 7o-v is quite labile in the presence of Lewis acid, hence the subsequent hydride abstraction step was unsuccessful. Overall, however, we can use this synthetic sequence to install phenyl onto a range of aryl halides and electronic arenes in a transition metal-free fashion with good efficiencies (Table 2).

Undoubtedly, the synthetic sequence in Table 2 is lengthy for the installation of a phenyl ring. The hydride abstraction step from cycloheptatrienes $\mathbf{6 / 7}$ to tropylium salts $\mathbf{1 / 8}$ is also not very atom economic. We envisioned that if we could form some intermediates being synthetically equivalent to $1 / 8$ in one step and carry out the oxidative ring contraction in the same reaction pot, the protocol would be more practical. Thus, we decided to explore this new approach by employing halotropylium halide 10 (Scheme 3a), which we previously used as halogenating or esterification/amidation reaction promoters. ${ }^{13 a-c}$ After an extensive reaction optimization study, we found out that bromotropylium bromide 10a was the best reagent for this purpose. A stoichiometric amount of 10a could react with the organometallic reagents from aryl halides $\mathbf{3}$ or react directly with arenes $\mathbf{4}$ to form intermediates 11. As the second bromine was able to dissociate off from the newly formed cycloheptatriene ring, $^{22} 11$ can serve a synthetic equivalent of tropylium salts $1 / 8$, eliminating the need for the hydride abstraction step. Subsequently, $\mathbf{1 1}$ was directly subjected to the oxidative ring contraction conditions developed in Table 1 to form the phenylated products (Scheme 3a).

Using this new one-pot protocol, we were able to convert a selected number of previously investigated aryl halides and arenes (3a-j and $\mathbf{4 k} \mathbf{k}-\mathbf{4 m}$ in Table 2, respectively) to their corresponding phenylated products ( $2 a-2 m$, Scheme 3a). The product yields of this new protocol were comparable to the overall yields of synthetic sequences in Table 2 . We also further investigated a range of new aryl and alkyl halide substrates, most of them worked efficiently with this formal phenylation procedure to give products $\mathbf{9 a - 9 i}$ in good yields. Most interestingly, this one-pot protocol was applicable to ketone substrates, which did not work with the synthetic sequence in Table 2. Indeed, acetophenone $\mathbf{4 0}$ and 4 '-chloroacetophenone $\mathbf{4 p}$ gave phenylated products $\mathbf{9 j}$ and $\mathbf{9 k}$, albeit in low yields. This type of a-phenylation reaction on carbonyl compounds is not straightforward and normally required transition metal-catalyzed or complex umpolung processes. ${ }^{23}$ 
Our oxidative ring contraction process could potentially be used in other applications than the formal phenylation reaction. For example, when we subjected suberenol $\mathbf{1 2}$ to similar reaction conditions (Scheme 3b), we observed an interesting shift in colors and photoluminescences of the solution. Suberenol 12 solution in acetonitrile is colorless under visible light but weakly light-blue luminescent under $365 \mathrm{~nm}$ UV irradiation. When an acid was introduced, a protonation and dehydration process occurred to generate a cationic dibenzosuberenylium species $\mathbf{1 3}$, which has similar reactivity to the tropylium ion. This solution immediately turned bright red under visible light and yellow luminescent under $365 \mathrm{~nm}$ UV light upon acidification. When being exposed to an oxidizing environment such as $\mathrm{H}_{2} \mathrm{O}_{2}, 13$ was oxidized to anthracene, and a number of other polyaromatic by-products, which instantly turned the solution colorless and green luminescent (Scheme 3b). Further studies to adapt this simple redox-sensitive system to sensing or imaging application of oxidants in biological environments are currently underway.
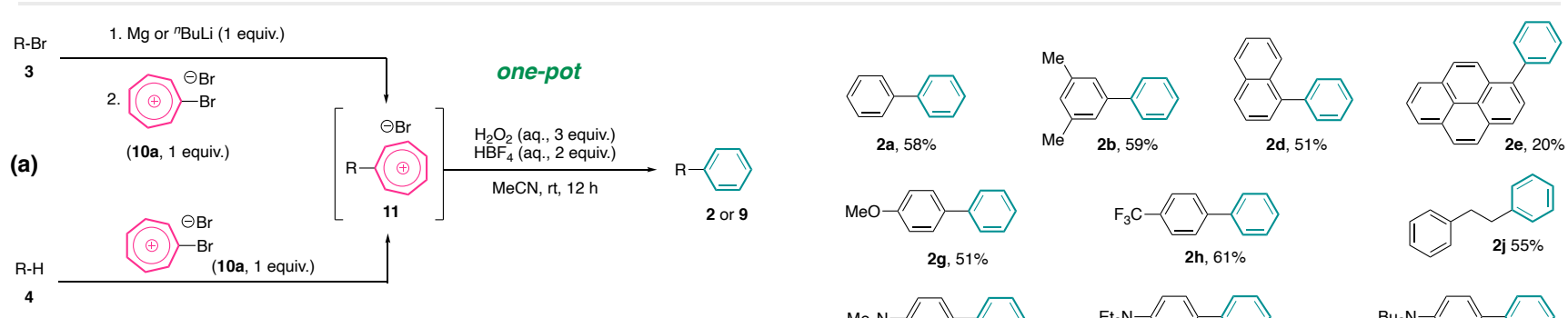

2g, $51 \%$

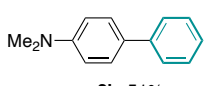

$2 k, 51 \%$
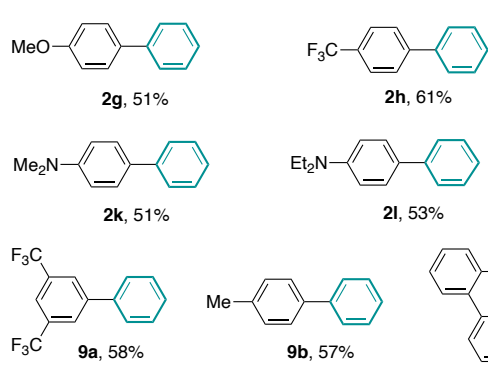

2d, $51 \%$
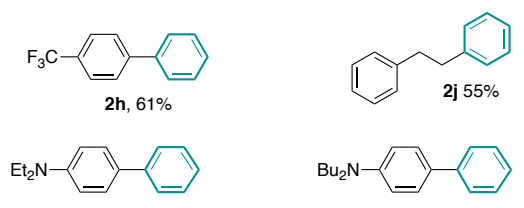

1, $53 \%$

$2 \mathrm{~m}, 51 \%$
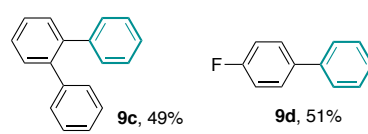

(b)
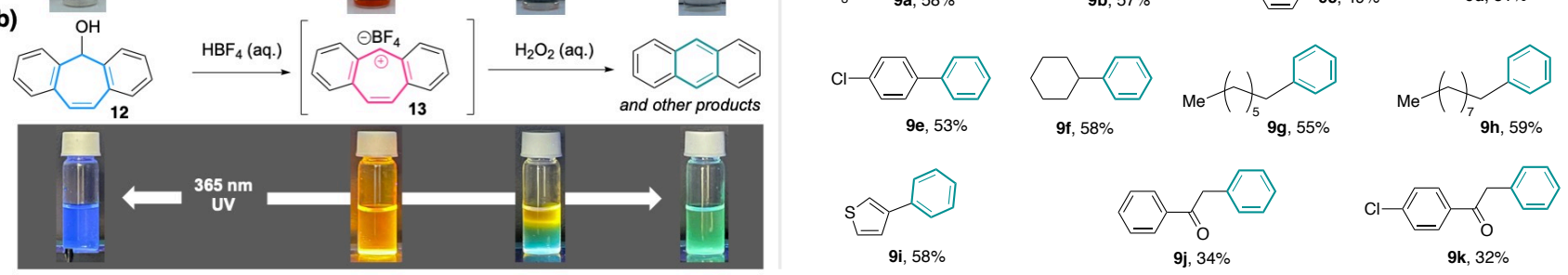

Scheme 3. One-pot formal phenylation reaction substrate scope.

In conclusion, we have developed a new protocol to allow formal phenylation reactions of aryl halides and electron-rich arenes in a transition-metal free manner. This protocol exploited the versatile electrophilicity and oxidizing ability of tropylium ion to construct the seven-membered ring framework and subsequently contract one carbon from that to produce the phenyl ring. It is also an interesting transformation at fundamental level in that the non-benzenoid aromatic tropylium ring is converted to the aromatic benzene ring. We are currently working on the incorporation of 
186 substituted tropylium ions onto organic structures and transforming them into polysubstituted 187 arylated frameworks and will report the outcomes in due course. 


\section{ASSOCIATED CONTENT}

190

191

192

193

194

\section{Supporting Information}

The Supporting Information is available free of charge: Experimental details and spectroscopic data for all products, full Gaussian reference, Cartesian coordinates, electronic and free energies.

\section{AUTHOR INFORMATION}

\section{Correspondence}

*T. V. N. E-mail: t.v.nguyen@unsw.edu.au (general enquiries)

*B. K. M. E-mail: binh.mai@pitt.edu (computational enquiries)

\section{CONFLICTS OF INTEREST}

There is no conflicts of interest to declare.

\section{ACKNOWLEDGMENTS}

This work was funded by the Australian Research Council (grant FT180100260 and DP200100063 to T. V. N).

\section{REFERENCES}

1. (a) I. Cepanec, Synthesis of Biaryls, Elsevier Science Ltd, Oxford, 2004; (b) M. C. Kozlowski, B. J. Morgan and E. C. Linton, Total synthesis of chiral biaryl natural products by asymmetric biaryl coupling. Chem. Soc. Rev. 2009, 38, 3193-3207.

2. F. Ullmann and J. Bielecki, Ueber Synthesen in der Biphenylreihe. Ber. Dtsch. Chem. Ges. 1901, $34,2174-2185$.

3. K. Tamao, K. Sumitani and M. Kumada, Selective carbon-carbon bond formation by crosscoupling of Grignard reagents with organic halides. Catalysis by nickel-phosphine complexes. J. Am. Chem. Soc. 1972, 94, 4374-4376. 
4. E. Negishi, A. O. King and N. Okukado, Selective carbon-carbon bond formation via transition metal catalysis. 3. A highly selective synthesis of unsymmetrical biaryls and diarylmethanes by the nickel- or palladium-catalyzed reaction of aryl- and benzylzinc derivatives with aryl halides. J. Org. Chem. 1977, 42, 1821-1823.

5. D. Milstein and J. K. Stille, A general, selective, and facile method for ketone synthesis from

8. (a) S. P. Stanforth, Catalytic cross-coupling reactions in biaryl synthesis. Tetrahedron 1998, 54, 263-303; (b) J. Hassan, M. Sévignon, C. Gozzi, E. Schulz and M. Lemaire, Aryl-Aryl Bond Formation One Century after the Discovery of the Ullmann Reaction. Chem. Rev. 2002, 102, 1359-1470; (c) D. Alberico, M. E. Scott and M. Lautens, Aryl-Aryl Bond Formation by Transition-Metal-Catalyzed Direct Arylation. Chem. Rev. 2007, 107, 174-238; (d) G. P. McGlacken and L. M. Bateman, Recent advances in aryl-aryl bond formation by direct arylation. Chem. Soc. Rev. 2009, 38, 2447-2464; (e) Y. Yang, J. Lan and J. You, Oxidative $\mathrm{C}-\mathrm{H} / \mathrm{C}-\mathrm{H}$ Coupling Reactions between Two (Hetero)arenes. Chem. Rev. 2017, 117, 8787-8863.

9. (a) C. Torborg and M. Beller, Recent Applications of Palladium-Catalyzed Coupling Reactions in the Pharmaceutical, Agrochemical, and Fine Chemical Industries. Adv. Synth. Catal. 2009, 351, 3027-3043; (b) J. Magano and J. R. Dunetz, Large-Scale Applications of Transition Metal-Catalyzed Couplings for the Synthesis of Pharmaceuticals. Chem. Rev. 2011, 111, 2177-2250; (c) C.-L. Sun and Z.-J. Shi, Transition-Metal-Free Coupling Reactions. Chem. Rev. 2014, 114, 9219-9280.

10. (a) N. E. Leadbeater and M. Marco, Transition-Metal-Free Suzuki-Type Coupling Reactions. Angew. Chem. Int. Ed. 2003, 42, 1407-1409; (b) N. E. Leadbeater and M. Marco, Transition-MetalFree Suzuki-Type Coupling Reactions: Scope and Limitations of the Methodology. J. Org. Chem. 
2003, 68, 5660-5667; (c) S. Yanagisawa, K. Ueda, T. Taniguchi and K. Itami, Potassium t-Butoxide

Alone Can Promote the Biaryl Coupling of Electron-Deficient Nitrogen Heterocycles and Haloarenes. Org. Lett. 2008, 10, 4673-4676; (d) W. Liu, H. Cao, H. Zhang, H. Zhang, K. H. Chung, C. He, H. Wang, F. Y. Kwong and A. Lei, Organocatalysis in Cross-Coupling: DMEDA-Catalyzed Direct C-H Arylation of Unactivated Benzene. J. Am. Chem. Soc. 2010, 132, 16737-16740; (e) C.-L. Sun, H.

Li, D.-G. Yu, M. Yu, X. Zhou, X.-Y. Lu, K. Huang, S.-F. Zheng, B.-J. Li and Z.-J. Shi, An efficient organocatalytic method for constructing biaryls through aromatic $\mathrm{C}-\mathrm{H}$ activation. Nature Chem. 2010, 2, 1044; (f) L. Xu, F.-Y. Liu, Q. Zhang, W.-J. Chang, Z.-L. Liu, Y. Lv, H.-Z. Yu, J. Xu, J.-J. Dai and H.-J. Xu, The amine-catalysed Suzuki-Miyaura-type coupling of aryl halides and arylboronic acids. Nature Catal. 2021, 4, 71-78.

11. (a) H. H. Wenk, M. Winkler and W. Sander, One Century of Aryne Chemistry. Angew. Chem. metal-free carbon-carbon and carbon-heteroatom bond-forming reactions using arynes. Chem. Soc. Rev. 2012, 41, 3140-3152; (c) C. M. Gampe and E. M. Carreira, Arynes and Cyclohexyne in Natural Product Synthesis. Angew. Chem. Int. Ed. 2012, 51, 3766-3778; (d) P. M. Tadross and B. M. Stoltz, A Comprehensive History of Arynes in Natural Product Total Synthesis. Chem. Rev. 2012, $112,3550-3577$.

12. (a) S. S. Babu, P. Muthuraja, P. Yadav and P. Gopinath, Aryldiazonium Salts in Photoredox Catalysis - Recent Trends. Adv. Synth. Catal. 2021, 363, 1782-1809; (b) D. R. Stuart, Aryl Transfer Selectivity in Metal-Free Reactions of Unsymmetrical Diaryliodonium Salts. Chem. Eur. J. 2017, 23, 15852-15863; (c) I. Ghosh, L. Marzo, A. Das, R. Shaikh and B. König, Visible Light Mediated Photoredox Catalytic Arylation Reactions. Acc. Chem. Res. 2016, 49, 1566-1577; (d) E. A. Merritt and B. Olofsson, Diaryliodonium Salts: A Journey from Obscurity to Fame. Angew. Chem. Int. Ed. 2009, 48, 9052-9070; (e) A. Roglans, A. Pla-Quintana and M. Moreno-Mañas, Diazonium Salts as Substrates in Palladium-Catalyzed Cross-Coupling Reactions. Chem. Rev. 2006, 106, 4622-4643.

13. (a) T. V. Nguyen and A. Bekensir, Aromatic Cation Activation: Nucleophilic Substitution of Alcohols and Carboxylic Acids. Org. Lett. 2014, 16, 1720-1723; (b) T. V. Nguyen and M. Hall, Activation of DMSO for Swern-type oxidation by 1,1-dichlorocycloheptatriene. Tetrahedron Lett. 2014, 55, 6895-6898; (c) T. V. Nguyen and D. J. M. Lyons, A novel aromatic carbocation-based coupling reagent for esterification and amidation reactions. Chem. Commun. 2015, 51, 3131-3134; (d) D. J. M. Lyons, R. D. Crocker, M. Blümel and T. V. Nguyen, Promotion of Organic Reactions by Non-Benzenoid Carbocylic Aromatic Ions. Angew. Chem. Int. Ed. 2017, 56, 1466-1484; (e) D. J. M. 
Lyons, R. D. Crocker, D. Enders and T. V. Nguyen, Tropylium salts as efficient organic Lewis acid catalysts for acetalization and transacetalization reactions in batch and flow. Green Chem. 2017, 3993-3996; (f) M. A. Hussein, V. T. Huynh, R. Hommelsheim, R. M. Koenigs and T. V. Nguyen, An efficient method for retro-Claisen-type $\mathrm{C}-\mathrm{C}$ bond cleavage of diketones with tropylium catalyst. Chem. Commun. 2018, 54, 12970-12973; (g) G. Oss, S. D. de Vos, K. N. H. Luc, J. B. Harper and T. V. Nguyen, Tropylium-Promoted Oxidative Functionalization of Tetrahydroisoquinolines. J. Org. Chem. 2018, 83, 1000-1010; (h) G. Oss, J. Ho and V. Nguyen Thanh, Tropylium Ion Catalyzes Pace, J. Ho and T. V. Nguyen, Tropylium-promoted carbonyl-olefin metathesis reactions. Chem. Sci. 2018, 9, 5145-5151; (j) R. D. Crocker, B. Zhang, D. P. Pace, W. W. H. Wong and T. V. Nguyen, Tetrabenzo[5.7]fulvalene: a forgotten aggregation induced-emission luminogen. Chem. Commun. 2019, 55, 11591-11594; (k) Y. Guo, T. V. Nguyen and R. M. Koenigs, Norcaradiene Synthesis via Visible-Light-Mediated Cyclopropanation Reactions of Arenes. Org. Lett. 2019, 21, 8814-8818; (I) M. A. Hussein, U. P. N. Tran, V. T. Huynh, J. Ho, M. Bhadbhade, H. Mayr and T. V. Nguyen, Halide Anion Triggered Reactions of Michael Acceptors with Tropylium Ion. Angew. Chem. Int. Ed. 2020, 59, 1455-1459; (m) D. J. M. Lyons, C. Empel, D. P. Pace, A. H. Dinh, B. K. Mai, R. M. Koenigs and T. V. Nguyen, Tropolonate Salts as Acyl-Transfer Catalysts under Thermal and Photochemical Conditions: Reaction Scope and Mechanistic Insights. ACS Catalysis 2020, 12596-12606; (n) K. Omoregbee, K. N. H. Luc, A. H. Dinh and T. V. Nguyen, Tropylium-promoted prenylation reactions of phenols in continuous flow. J. Flow. Chem 2020, 10, 161-166; (o) C. Empel, T. V. Nguyen and R. M. Koenigs, Tropylium-Catalyzed O-H Insertion Reactions of Diazoalkanes with Carboxylic Acids. Org. Lett. 2021, 23, 548-553; (p) D. J. M. Lyons, R. D. Crocker and T. V. Nguyen, Stimuli-Responsive Organic Dyes with Tropylium Chromophore. Chem. Eur. J. 2018, 24, 10959-10965.

14. A. Tamoto, N. Aratani and H. Yamada, Contraction of $\pi$-Conjugated Rings upon Oxidation from Cyclooctatetraene to Benzene via the Tropylium Cation. Chem. Eur. J. 2017, 23, 1638816392.

15. (a) M. E. Volpin, D. N. Kursanov and V. G. Dulova, Tropilium salts and benzene. Tetrahedron 1960, 8, 33-37; (b) S. Kobayashi, T. Tezuka and W. Ando, A novel conversion of tropylium ion to benzenoid compounds in the reaction with peroxide ion. Tetrahedron Lett. 1979, 20, 261-262.

16. (a) C. R. Solorio-Alvarado, Y. Wang and A. M. Echavarren, Cyclopropanation with Gold(I) Carbenes by Retro-Buchner Reaction from Cycloheptatrienes. J. Am. Chem. Soc. 2011, 133, 1195211955; (b) P. R. McGonigal, C. de León, Y. Wang, A. Homs, C. R. Solorio-Alvarado and A. M. 
Echavarren, Gold for the Generation and Control of Fluxional Barbaralyl Cations. Angew. Chem. Int. Ed. 2012, 51, 13093-13096; (c) Y. Wang, P. R. McGonigal, B. Herlé, M. Besora and A. M. Echavarren, Gold(I) Carbenes by Retro-Buchner Reaction: Generation and Fate. J. Am. Chem. Soc. 2014, 136, 801-809; (d) Y. Wang, M. E. Muratore, Z. Rong and A. M. Echavarren, Formal (4+1) Cycloaddition of Methylenecyclopropanes with 7-Aryl-1,3,5-cycloheptatrienes by Triple Gold(I) Catalysis. Angew. Chem. Int. Ed. 2014, 53, 14022-14026; (e) S. Ferrer and A. M. Echavarren, Synthesis of Barbaralones and Bullvalenes Made Easy by Gold Catalysis. Angew. Chem. Int. Ed. 2016, 55, 11178-11182; (f) B. Herlé, P. M. Holstein and A. M. Echavarren, Stereoselective cisVinylcyclopropanation via a Gold(I)-Catalyzed Retro-Buchner Reaction under Mild Conditions. ACS Catalysis 2017, 7, 3668-3675; (g) X. Yin, M. Mato and A. M. Echavarren, Gold(I)-Catalyzed Synthesis of Indenes and Cyclopentadienes: Access to ( \pm )-Laurokamurene B and the Skeletons of the Cycloaurenones and Dysiherbols. Angew. Chem. Int. Ed. 2017, 56, 14591-14595; (h) M. Mato, B. Herlé and A. M. Echavarren, Cyclopropanation by Gold- or Zinc-Catalyzed Retro-Buchner Reaction at Room Temperature. Org. Lett. 2018, 20, 4341-4345; (i) M. Mato and A. M. Echavarren, Donor Rhodium Carbenes by Retro-Buchner Reaction. Angew. Chem. Int. Ed. 2019, 58, 2088-2092; (j) M. Mato, C. García-Morales and A. M. Echavarren, Generation of Gold(I) Carbenes by RetroBuchner Reaction: From Cyclopropanes to Natural Products Synthesis. ChemCatChem 2019, 11, 53-72.

17. (a) Z. Chen, C. S. Wannere, C. Corminboeuf, R. Puchta and P. v. R. Schleyer, NucleusIndependent Chemical Shifts (NICS) as an Aromaticity Criterion. Chem. Rev. 2005, 105, 3842-3888; (b) C. Corminboeuf, T. Heine, G. Seifert, P. v. R. Schleyer and J. Weber, Induced magnetic fields in aromatic [n]-annulenes-interpretation of NICS tensor components. Phys. Chem. Chem. Phys. 2004, 6, 273-276; (c) L. J. Karas, A. T. Campbell, I. V. Alabugin and J. I. Wu, Antiaromaticity Gain Activates Tropone and Nonbenzenoid Aromatics as Normal-Electron-Demand Diels-Alder Dienes. Org. Lett. 2020, 22, 7083-7087.

18. See the Supporting Information for further details.

19. DFT calculations were performed at the M06-2X/6-311+G(2d,2p)/SMD(MeCN)//M06-2X/6$31+G(d, p) / S M D(M e C N)$ level of theory. See the Computational Supporting Information for computational details.

20. (a) R. D. Bach and M.-D. Su, The Transition State for the Hydroxylation of Saturated Hydrocarbons with Hydroperoxonium Ion. J. Am. Chem. Soc. 1994, 116, 10103-10109; (b) Å. M. 
Leere Øiestad, A. C. Petersen, V. Bakken, J. Vedde and E. Uggerud, The Oxidative Power of Protonated Hydrogen Peroxide. Angew. Chem. Int. Ed. 2001, 40, 1305-1309; (c) C. Adlhart, O.

Sekiguchi and E. Uggerud, On the Gas-Phase Reactivity of Complexed $\mathrm{OH}+$ with Halogenated Alkanes. Chem. Eur. J. 2005, 11, 152-159.

21. P. Mueller and J. Rocek, Oxidation of hydroaromatic systems. IV. Chromic acid oxidation of cycloheptatriene. J. Am. Chem. Soc. 1974, 96, 2836-2840.

22. (a) B. Föhlisch, P. Bürgle and D. Krockenberger, Zur Chemie cyclischer $\pi$-Elektronensysteme, IV. Stabile Chinotropilidene. Chem. Ber. 1968, 101, 2717-2730; (b) C. Jandl, D. C. Mayer and A. Pöthig, The Ambivalent Nature of Halogenated Tropone Derivatives: Dihalocycloheptatriene vs. Halotropylium Halide. Eur. J. Org. Chem. 2017, 2017, 4255-4259.

23. (a) T. Miyoshi, T. Miyakawa, M. Ueda and O. Miyata, Nucleophilic $\alpha$-Arylation and $\alpha$-Alkylation of Ketones by Polarity Inversion of N-Alkoxyenamines: Entry to the Umpolung Reaction at the $\alpha$ Carbon Position of Carbonyl Compounds. Angew. Chem. Int. Ed. 2011, 50, 928-931; (b) D. FelipeBlanco and J. C. Gonzalez-Gomez, Salicylic Acid-Catalyzed Arylation of Enol Acetates with Anilines. Adv. Synth. Catal. 2018, 360, 2773-2778; (c) E. Picazo, S. M. Anthony, M. Giroud, A. Simon, M. A. Miller, K. N. Houk and N. K. Garg, Arynes and Cyclic Alkynes as Synthetic Building Blocks for Stereodefined Quaternary Centers. J. Am. Chem. Soc. 2018, 140, 7605-7610; (d) J. Li, A. Bauer, G. Di Mauro and N. Maulide, $\alpha$-Arylation of Carbonyl Compounds through Oxidative C-C Bond Activation. Angew. Chem. Int. Ed. 2019, 58, 9816-9819; (e) W.-H. Huang, G.-B. Huang, W.-R. Zhu, J. Weng and G. Lu, Transition metal-free synthesis of $\alpha$-aryl ketones via oxyallyl cation capture with arylboronic acids. Org. Chem. Front. 2020, 7, 2480-2485; (f) G. M. Kiefl and T. Gulder, $\alpha-$ Functionalization of Ketones via a Nitrogen Directed Oxidative Umpolung. J. Am. Chem. Soc. 2020, 142, 20577-20582; (g) W. Zawodny, C. J. Teskey, M. Mishevska, M. Völkl, B. Maryasin, L. González and N. Maulide, $\alpha$-Functionalisation of Ketones Through Metal-Free Electrophilic Activation. Angew. Chem. Int. Ed. 2020, 59, 20935-20939. 